\title{
Ethical Considerations in Human Movement Research
}

\begin{abstract}
Steve Olivier
In the past decade, ethical issues in research involving human subjects have exploded into the public consciousness. In reviewing past human subject abuse, it is evident that, in human experimentation, legislation has not been sufficient to curb excesses. Selected journal reviews indicate that informed consent is often not reported for studies where such consent is deemed appropriate. This does not necessarily mean that consent was not obtained, or that subjects were abused or exploited. It does, however, introduce the possibility that many researchers either are not cognizant of, or merely pay lip service to, the principles that form a code of ethics. Ethics in research involving human subjects is not a settled issue. Researchers ought to be aware that the principles they accept may be less conclusive, and the guidelines they apply may be less protective, than such principles and guidelines appear to be. Testing human subjects is not a right, but a privilege, and the rights of the subject ought to outweigh the desires of the researcher to conduct research.
\end{abstract}

As in other areas of scientific inquiry, there has been an increasing demand for research to be undertaken in the subdisciplines of human movement studies. With the increase in research, and particularly as a result of problems arising in the medical arena, ethical issues have recently exploded into the public consciousness, so much so that the ethical review of research on human beings can be considered a growth industry (Pettit, 1992). Progress in human movement studies and in biomedical research has demanded that human subjects be increasingly subjected to manipulative, and sometimes even invasive procedures. Research per se is concerned with (usually) novel techniques used to develop or contribute to generalizable knowledge (Veatch, 1989), and this means that while procedures may be carefully implemented and controlled, the very nature of research means that the specific effects cannot be predetermined.

In this paper, issues that ought to be considered by researchers who use human subjects are highlighted. The principles that form the construct of a code of research ethics are identified, and past human experimentation and current research in human movement studies are evaluated against this construct. In

Steve Olivier is with the Department of Human Movement Science at the University of Zululand, Private Bag X1001, Kwa-Dlangezwa 3886, South Africa. 
doing this, the efficacy of both legislation and self-regulation is examined. Particular attention is paid to the context in which informed consent operates, and an argument against overregulation of research is reviewed. Given the context within which research in human movement studies operates, it is incumbent on researchers, supervisors, publishers, and ethical review boards to consider the ethical issues raised in human experimentation.

\section{Abuse of Human Subjects}

Between 1930 and 1945 Japan conducted experiments on prisoners of war at a research installation named Unit 731, near Harkin. Veatch (1989) reported that the facility was capable of producing eight tons of bacteria per month, and that experiments on humans included prolonged exposure of the liver to x-rays, freezing body parts to try various methods of thawing, pumping the body full of horse blood, and vivisection. Also, experiments were conducted on the human response to anthrax, botulism, cholera, dysentery, smallpox, syphilis, typhoid, and typhus. Officials of the United States adopted a baldly utilitarian standpoint upon discovery of the unit and its activities. The officials protected the researchers from prosecution and justified this on the grounds that the value of the information far outweighed the value of prosecution, as the findings greatly augmented scientific knowledge. Besides, the officials reasoned, such findings were unobtainable elsewhere due to more stringent controls on human subject research. This rather extreme example raises the issue of conflict between moral principles and self-interest on the part of the researcher(s).

In 1932 the U.S. Public Health Service commenced a study that involved monitoring the condition of untreated syphilis in a population of rural black males near Tuskegee, Alabama. The subjects were kept ignorant of the experiment and were actively discouraged from seeking or receiving effective treatment, lest that interfere with the data (Veatch, 1989). This study was only terminated in 1972. The primary issue raised here is that of gross abuse of research subjects - the investigators manifested a total disrespect for the subjects as persons.

Again, the example is an extreme one, and it could be argued that today's moral climate renders a repeat unlikely. However, acceptance of such an argument should be approached with caution. Society continues to allow and encourage human experimentation, and legislation is not sufficient to curb excesses where information is demanded. Caplan (1992) noted that it is often presumed that those who know what is ethical will not behave in immoral ways, but he points out that this is not necessarily so. Regulations on medical ethics in Germany prior to World War II were detailed and stringent, yet they did not prevent abuses from occurring in prisoner of war camps, illustrating that neither official endorsement nor high aspirations are enough to ensure that a code of ethics will protect subjects.

Pettit (1992), in taking issue with the way institutional review boards function, agrees that merely formalizing ethical practices may not be enough, contending that self-regulation is the most effective safeguard against the abuse of subjects. Ethics in research involving human subjects is not a settled issue involving detailed statutory and regulatory requirements and procedures. The history already reviewed indicates that any such claim would need to be viewed 
skeptically, as the principles we accept may be less conclusive, and the guidelines we apply may be less protective, than they appear to be (Veatch, 1989). Constant review seems to be a prerequisite for research involving human subjects, with such research being justified by appealing to ethical principles.

\section{Ethical Principles}

The identification of those principles is necessary for discussion to proceed. Rifkin (cited in "Ethics in Embryo," 1987) contends that Western medical science has moved and is still moving toward utilitarianism and that this process has occurred in tandem with a gradual devaluing of life. On the other hand, Brodie and Stopani (1990) state that current societal opinion reflects the present ethical belief that it is more important to avoid risk to a subject than to gain future benefit or advance knowledge. Which viewpoint holds sway, and more importantly, which viewpoint ought to carry more weight? The allied question of research perhaps ranking consequentialist principles over nonconsequentialist ones needs to be addressed.

At the beginning of Western moral philosophy, Plato (1987) recognized the tension between self-interest and virtue. There is perhaps a need to examine whether research in human movement studies generally reflects "bottom-line", ethics (which is concerned only with results) or virtue ethics (which is also concerned with conduct). The notion that there is an inevitable collision between maximizing benefit and subject autonomy thus needs to be addressed. The principle of autonomy also needs to be discussed in some detail, along with allied issues such as possibly justifying paternalism and deception against violation of the principles of autonomy and veracity.

Consideration of questions such as those posed above leads to the broad question of what principles a code of ethics for research involving human subjects ought to embrace. Here one needs to examine general principles such as respect for persons, beneficence, nonmaleficence, justice, veracity, fidelity, privacy, confidentiality, and universalizability. Also worthy of examination are more specific elements of codes of ethics such as consideration of cultural factors, nondiscrimination, sanctions against offenders, compliance with procedures, and reports of violations.

\section{Current Research}

Having tentatively suggested some principles that codes of ethics in human movement studies perhaps ought to embrace, I will now move to an exploratory evaluation of research in the field in terms of these principles. A preliminary examination of journal reviews reveals that many researchers either are not cognizant of or merely pay lip service to the principles that form a code of ethics. Pettit (1992) reports that in 1966 Henry Beecher of Harvard Medical School published a survey of ethical behavior in clinical research in the New England Journal of Medical Research. After examining the major journals on clinical research, Beecher found 50 examples of ethically dubious research on human subjects. Consent was mentioned in only 2 of these articles. 
More recently, Brodie and Stopani (1990), in a survey of just one journal, found that for those studies for which it was appropriate, informed consent was obtained for only 14 out of 81 papers. In South Africa, an examination of papers published in the S.A. Journal for Research in Sport, Physical Education and Recreation reveals that informed consent was reported in only 13 out of 94 papers for which such consent was deemed appropriate.

Papers published in the Ergonomics South Africa Journal show a similar trend. During the period July 1989 to July 1993, 20 papers were published for which obtaining informed consent was deemed necessary by this author. Of these, only one author reported that written informed consent had been obtained. On the positive side, seven authors reported that subjects were volunteers. ${ }^{1}$ Of course, these results do not necessarily mean that consent was not obtained, or that subjects were abused or exploited. These results merely indicate that consent was not reported. The potential for abuse, however, exists, and "we must be aware of the rights of subjects and not take the expedient route to conduct our research" (Zelaznik, 1993, p. 65).

Again, while not indicating abuse, the reviews above introduce the possibility that many researchers either are not cognizant of, or merely pay lip service to, the principles of a code of ethics. From this one could conclude that insufficient attention is being paid to this controversial yet necessary facet of research ethics. Perhaps the researchers or their defenders would counter that consent was obtained but was not reported in the manuscripts. This however will not do. First, the ethics of the omission are questionable, and second, such omission violates the fundamental requirement that experiments be recorded in sufficient detail to enable replication by others.

From the above one can see that obtaining informed consent is often not reported. What then about the other elements of the proposed ethical construct? In the following review of recent research I will highlight examples where abuses of principles of research ethics may occur. ${ }^{2}$

Wagner (1991) points out that ergogenic aids such as amphetamines and anabolic-androgenic steroids have the potential to produce a wide array of adverse physiological and psychological effects, and he states the following:

Whether the ergogenic aids are real or perceived, the potential for adverse effects exists ... [ [and] potential health complications represent a serious risk to an otherwise healthy population. (p. 251)

Bahrke, Yesalis, and Wright (1990) concur and place additional emphasis on the issue of psychological dependence on such aids. Athletes and research subjects (who are often athletes) are clearly not immune to factors that contribute and predispose one to drug abuse in the general population. Wagner (1991) in fact contends that factors unique to athletes may place them at an increased risk for drug abuse. Given the adverse effects and the possibility of dependence and the fact that there is a substantial body of research in this area, there is a need to question what conditions, if any, justify the administration of steroids to research subjects.

It is not reported whether Crist, Stackpole, and Peake (1983) considered this question when administering relatively high doses of testosterone cypionate and nandrolone decanoate to nine volunteer subjects in an effort to determine 
the effects of androgenic-anabolic steroids on neuromuscular power and body composition. Although no significant effects were noted, the subjects reported subjective feelings of increased strength after the administration of anabolic agents. These subjective impressions may be an important factor in the acceptance of steroids by athletes in attempts to improve physical performance. Furthermore, such impressions may then result in psychological dependence, with the immediate benefits being readily visible, while the longer term adverse effects are not yet apparent.

Although not as overtly dramatic as the effects of steroids, nicotine is a complex addictive drug that has been shown to alter many of the body's regulatory mechanisms (Marks \& Perkins, 1990). Marks and Perkins stated that the health hazards of smoking are clearly evident and that tobacco withdrawal syndrome occurs within 24 hours of abstinence and can result in headaches, constipation, irritability, and fatigue. They reviewed several studies involving the administration of nicotine by various means to both smokers and nonsmokers. As with some other research in human movement studies, such research needs to be examined against the proposed construct of ethical principles mentioned earlier. Also, the question of alternative avenues of research, using humans only as a last resort, needs to be raised.

Another ergogenic aid that has increasingly become the focus of research is the process of blood-doping (erythrocemia). Jones and Tunstall-Pedoe (1989) noted that evidence suggests that blood doping can result in significant improvements in physiological variables such as maximum oxygen uptake and lactate buffering, such changes matching improvements in endurance performance. This is supported by the research of Robertson et al. (1984), who found that maximal $\mathrm{VO}_{2}$ and physical work capacity increased in women following induced erythrocemia.

As with other research involving ergogenic aids, studies involving induced erythrocemia may present some ethical problems. First, there is the possibility of adverse effects as a result of the procedure. Besides the theoretical risks of transfer of infectious diseases such as AIDS and hepatitis if heterologous transfusion is used, any intravenous infusion carries risks such as venous thrombosis, phlebitis, septicemia, and even pulmonary embolism. Also, human recombinant erythropoietin is now available and has the potential to produce erythrocemia similar to or greater than blood doping. However, erythropoietin use can lead to hypertension, heart failure, or strokes, and although not conclusively linked, several deaths of athletes known to have been taking the substance have been reported (see Wagner, 1991).

Second, having experienced the benefits of the procedure firsthand, a subject is presented with the choice of whether to continue with the practice on his or her own. Given the increasingly competitive nature of sport and the attendant rewards for performance, the athlete is placed a step closer towards having a moral problem. It will not do to argue that the problem existed anyway.

As a research subject, the athlete has, as it were, been "introduced" to the problem. Given that blood doping is banned by the International Olympic Committee, the individual who chooses to continue has opted to operate outside the code of ethics adopted by the duly constituted authorities. The argument thus is that by virtue of being a research subject, albeit voluntarily, the individual has been placed a step closer to temptation as a result of inadequate consideration 
of ethics by the researcher. This is, of course, not to suggest a utopian vision of research as being totally risk free. Rather, it means that it would be dangerous for us as researchers to assume that increasing general concern with ethical issues and the rights of subjects in the present-day moral climate necessarily means that such issues have been considered, even if such consideration is not explicitly reported.

Wolfe et al. (1989) noted that during intensive exertion by pregnant women, maternal skeletal muscle and the fetus may compete for blood flow, oxygen delivery, and essential fuel substrates, with the attendant hypothetical risks of acute fetal hypoxia, hyperthermia, and malnutrition. With repeated chronic exercise, fetal growth retardation and altered fetal development may result. Although there are postulated benefits of exercise during pregnancy, the authors noted that these remain to be confirmed (Wolfe et al., 1989, p. 274). Given the dangers outlined above, it would seem prudent to proceed cautiously when requiring pregnant participants to adhere to an exercise regime.

Despite the potential problems, extensive research is performed utilizing pregnant women as subjects. For example, Clapp, Wesley, and Sleamaker (1989) investigated thermoregulatory and metabolic responses to jogging prior to and during pregnancy. While acknowledging that in such studies ethical and regulatory concerns limit protocol, the authors concluded that exercise conforming to the type and intensity and duration of that of their study may indeed limit fetal substrate availability in late pregnancy. While findings such as these may icrease knowledge in this area, the methods used may violate the autonomy of the participants (both mother and fetus) and may be maleficent to subjects. The general question again raised is whether, in research involving human participants, utility should trump the right to self-determination of subjects.

\section{Informed Consent}

"Informed consent" is a much-debated issue, with critics claiming that, as generally understood and applied, it offers inadequate protection to human research subjects. Others may counter that subjects are, at present, infinitely better protected than they were in the past and that the imperfections of the concept should not necessarily result in discarding the process (Wheeler, 1991).

Before continuing, I will briefly examine what is meant by informed consent. Mahon (1987) states that consent can be considered "informed" when "it is given in the full, or clear, realization of what the tests involve, including an awareness ... of risk attached to what takes place" (p. 203). According to Zelaznik (1993), "subjects must be fully informed of the risks, procedures, and potential benefits, and that they are free to end their participation in the study with no penalty whatsoever"' (p. 63). Informed consent has also been defined as "the knowing consent of an individual . . . able to exercise free power or choice without inducement or any element of force, fraud, deceit, duress, or other form of constraint or coercion"' ("Policy Statement," 1990, p. vi).

When should informed consent be obtained? A policy statement in Medicine and Science in Sports and Exercise holds that "any experimental subject or clinical patient who is exposed to possible physical, psychological, or social injury must give informed consent prior to participating in a proposed project", 
("Policy Statement,"' 1990, p. vi). Furthermore, the journal requires that all appropriate steps be taken in obtaining the informed consent of any and all human participants employed by investigators submitting manuscripts for review and possible publication, and authors are required to indicate that consent was obtained. Given the review results reported earlier, perhaps the South African Federation for Movement and Leisure Sciences and the Ergonomics Society of South Africa should institute a similar publication requirement.

An important issue with regard to current research in human movement studies is whether obtaining informed consent is largely ceremonial. As mentioned earlier, there are indications that it is sometimes not even ceremonial and that in a large number of cases it is either not reported (a serious omission in its own right) or is not obtained. The question that follows from this is whether there is a meaningful exchange of information when the consent process takes place. With participation the key to informed consent, it is necessary to ask whether the proper climate exists for the communication process in the informed consent context (Veatch, 1989, p. 186). This may be of particular importance in multicultural research environments such as South Africa, where an examination of the necessity to have the facility to exchange information multilingually, both verbally and in writing, may be advisable. Further, the autonomy of subjects is again at issue here, particularly when researchers utilize "captive" populations such as students, tournament participants, prisoners, and inmates. Here the question changes from how informed subjects are to how free they are, and whether some form of coercion occurs.

\section{Institutional Review Boards and Scientific Progress}

Earlier I noted that the increasing demand for information from research has necessitated manipulative and invasive procedures, and I have strongly advocated the need to focus on ethical issues in conducting such research. Some researchers may bemoan the ascendancy of individual rights, contending that abuses do not occur and that knowledge would not advance without an element of uncertainty or risk. Pettit (1992) shares the latter concerns, and questions whether blind adherence to the "growth industry" of research ethics could retard progress. He contends that ethical review (as it currently stands and the way it is heading) is endangering valuable research on human beings and, moreover, is endangering the very ethic that is needed to govern that research. He argues that the current reactive dynamic to ethical abuses in research may lead to a serious reduction in the current scope of research and to a substantial compromise of the ethic that currently governs research practice. Ethics committees, he holds, with no rewards for good decisions and severe penalties for bad ones, are becoming more conservative and restrictive, and this not only eliminates much good research, but also may lead to a deterioration in the commitment of researchers to the ethic which currently prevails.

Pettit's views support those of Mosher (1988), who contends that institutional review boards are encouraging a growing bureaucracy that inhibits science by reducing creative nonconformity, and that scientific progress is being slowed or prevented by institutional review boards. This may be so, and researchers need to guard against practices that restrict attempts to advance knowledge merely because such practices are fashionable. 
In short, researchers need to justify those practices, and I would argue that the justification lies in the fact that it is naive to assume that the best protection for research subjects lies in the conscience of the investigator, given the previous allusion to the potential conflict between moral principles and self-interest. Earlier it was noted that stringent legislation by itself provides no foolproof safeguards against ethical malpractice. On the other hand, it should be acknowledged that the existence of procedural safeguards has done much to legitimate the place of human research in society. It could be argued that there is a human tendency to overrate the benefits and underestimate the risks of research. Recognition and acceptance of such a trend would serve to justify the questions posed by this paper, as well as justify setting more stringent ethical requirements and imposing additional safeguards.

Although I acknowledge the rights of researchers and accept a "progress imperative" view of science, neither legislation nor self-awareness are sufficient conditions to prevent malpractice. Zelaznik (1993) maintains that testing human subjects is not a right, but a privilege, and it is therefore incumbent on the researcher to maintain the rights of participants in order to maintain their participation. On this view, researchers should consider the moral stance that the rights of the study participant ought always to outweigh the desires of the researcher to conduct research.

\section{References}

Bahrke, M.S., Yesalis, C.E., \& Wright, J.E. (1990). Psychological and behavioural effects of endogenous testosterone levels and anabolic-androgenic steroids among males: A review. Sports Medicine, 10, 303-337.

Brodie, D.A., \& Stopani, K. (1990). Experimental ethics in sports medicine research. Sports Medicine, 9, 143-150.

Caplan, A.L. (1992). How did medicine go so wrong? In A.L. Caplan (Ed.), When medicine went mad (pp. 53-92). New York: Humana Press.

Clapp, J.F., Wesley, M., \& Sleamaker, R.H. (1987). Thermoregulatory and metabolic responses to jogging prior to and during pregnancy. Medicine and Science in Sports and Exercise, 19, 124-130.

Cooper, C.J., \& Noakes, T.D. (1994). Psychiatric disturbances in users of anabolic steroids. South African Medical Journal, 84, 509-512.

Crist, D.M., Stackpole, P.J., \& Peake, G.T. (1983). Effects of androgenic-anabolic steroids on neuromuscular power and body composition. Journal of Applied Physiology, 54, 366-370.

Ethics in embryo: A symposium. (1987, September). Harpers Magazine, 3, 57-63.

Jones, M., \& Tunstall-Pedoe, D.S. (1989). Blood doping-a literature review. British Journal of Sports Medicine, 23(2), 84-88.

Mahon, J. (1987). Ethics and drug testing in human beings. In J.D.G. Evans (Ed.), Moral philosophy and contemporary problems (pp. 199-211). Cambridge: Press Syndicate of the University of Cambridge.

Marks, B.L., \& Perkins, K.A. (1990). The effects of nicotine on metabolic rate. Sports Medicine, 10, 277-285.

Mosher, D.L. (1988). Balancing the rights of subjects, scientists and society: 10 principles of human subject committees. The Journal of Sex Research, 24, 378-385. 
Plato. (1987). The republic (Desmond Lee, Trans., ed.). London: Penguin Books.

Pettit, P. (1992). Instituting a research ethic: Chilling and cautionary tales. Bioethics, 6, 89-112.

Policy Statement. (1990). Medicine and Science in Sports and Exercise, 22, vi.

Robertson, R.J., Gilcher, R., Metz, K.F., Caspersen, C.J., Allison, T.G., Abbot, R.A., Skrinar, G.S., Kranse, J.R., \& Nixon, P.A. (1984). Haemoglobin concentration and aerobic work capacity in women following induced erythrocemia. Journal of Applied Physiology, 57, 568-575.

Veatch, R.M. (Ed.) (1989). Medical ethics. Boston: Jones \& Bartlett.

Wolfe, L.A., Hall, P., Webb, K.A., Goodman, L., Monga, M., \& McGrath, M.J. (1989). Prescription of aerobic exercise during pregnancy. Sports Medicine, 8, 273-301.

Wagner, J.C. (1991). Enhancement of athletic performance with drugs: An overview. Sports Medicine, 12, 250-265.

Wheeler, D.L. (1991, December 4). Informed consent questioned in research using humans. The Chronicle of Higher Education, p. A14.

Zelaznik, H.N. (1993). Ethical issues in conducting and reporting research: A reaction to Kroll, Matt, \& Safrit. Quest, 45, 62-68.

\section{Notes}

${ }^{1}$ For the purposes of this specific journal review consent was considered necessary for research utilizing captive populations such as students, schoolchildren, and tournament participants, where subtle forms of coercion may operate even if not intended. Also, in research concerning minors, it was deemed necessary to obtain parental informed consent.

${ }^{2}$ Articles cited in this review serve as examples. This is not to suggest that the authors did not consider the ethical issues pertaining to their research, merely that they did not report on those considerations. Of course, publication pressures, such as available space, may deter authors from such reporting. 